\title{
Turbulent temperature profile in the quasi-fully developed region of a micro-tube
}

\author{
Yutaka ASAKO* and Chungpyo HONG** \\ *Department of Mechanical Precision Engineering \\ Malaysia-Japan International Institute of Technology \\ University Technology Malaysia \\ Jalan Sultan Yahya Petra, 54100 Kuala Lumpur, Malaysia \\ E-mail: y.asako@utm.my \\ **Department of Mechanical Engineering, Kagoshima University \\ 1-21-40 Korimoto, Kagoshima, 890-8580, Japan
}

Received: 8 August 2016; Revised: 24 September 2016; Accepted: 30 April 2017

\begin{abstract}
Turbulent temperature profiles in the quasi-fully developed region of a micro-tube were obtained numerically by solving the energy equation including the substantial derivative of pressure and viscous dissipation terms for the case of constant wall temperature. The fluid was assumed to be an ideal gas with constant density over the cross-section. The turbulent velocity profile was approximated by the three-layer model of Von Kármán. The temperature profiles were compared with the laminar flow and previous numerical solutions. The total temperature was higher than the wall temperature depending on the Mach number. The static temperature in the quasi-fully developed region agrees well with the temperature results for an ideal gas flow obtained by solving the Navier-Stokes and energy equations.
\end{abstract}

Key words: Temperature profile, Turbulent flow, Quasi-developed region, Energy equation, Viscous dissipation, Substantial derivative of pressure

\section{Introduction}

Design and fabrication of Micro Electro Mechanical Systems (MEMS) have increased the need for understanding of fluid flow and heat transfer in micron-sized channels. The transverse velocity gradient for flow in micron-sized channel is quite high and results in large pressure drop. For the case of gas flow in micro-tube, the flow accelerates even far away from the entrance and therefore, it does not become fully developed. However, the changes in the flow direction are small compared with the radial direction and the velocity in the $r$-direction can be assumed to be zero. The region where the axial velocity and temperature gradients (e.g. $\partial u / \partial x, \partial T / \partial x$ ) and the radial velocity are small and also the density can be considered constant is called quasi-fully developed region (Asako et al., 2003).

The effect of viscous heat dissipation is significant in a micro-tube. Therefore, the energy equation including the viscous dissipation (VD) and the substantial derivative of pressure (SDP) terms should be solved to obtain the temperature profile in the quasi-fully developed region (e.g. Asako, 2014). A careful search of the literature failed to disclose any prior work on the determination of the temperature profile in the quasi-fully developed region of a tube with the exception of Asako and Hong (2017) who obtained the temperature profiles for laminar slip and nonslip flows in the quasi-fully developed region of a tube. This has motivated the present study to obtain the temperature profile of turbulent flows in a quasi-fully developed region of a tube.

In this study, the turbulent temperature profiles in the quasi-fully developed region are obtained by solving the energy equation including the SDP and the VD terms. The fluid is assumed to be an ideal gas with constant density over the cross-section. The three-layer universal velocity model of Von Kármán (Von Kármán, 1939 or e.g. Mills, 1992) was used for the velocity profile. The discretized energy equation is solved numerically to obtain the temperature 
profiles. The temperature profiles are compared with the results for laminar flow and also with the previous numerical solutions.

\section{NOMENCLATURE}

$C_{f}$

$C_{p}$

$d$

$h$

$k$

$M a$

$\dot{m}$

$\mathrm{Pr}$

$p$

$P$

$\dot{Q}$

Re

$R_{\text {gas }}$

$T$

$u$

$U$

$u^{+}$

$X, R$

$r_{0}{ }^{+}$

Y

y

$y^{+}$

$\beta$

$\gamma$

$\lambda$

$\mu$

$v$

$\theta$

$\rho$

Subscript

ave

eff

$T$

in

$w$

Superscript

friction coefficient

specific heat at constant pressure, $\mathrm{J} /(\mathrm{kg} \mathrm{K})$

tube diameter, $\mathrm{m}$

specific enthalpy, $\mathrm{J} / \mathrm{kg}$

turbulent energy, $\mathrm{m}^{2} / \mathrm{s}^{2}$

Mach number

mass flow rate, $\mathrm{kg} / \mathrm{s}$

Prandtl number

static pressure, $\mathrm{Pa}$

dimensionless pressure

heat transfer rate, $\mathrm{W}$

Reynolds number

gas constant, J/(Kg K)

temperature, $\mathrm{K}$

velocity, $\mathrm{m} / \mathrm{s}$

dimensionless velocity

dimensionless velocity based on wall shear stress

dimensionless coordinates

dimensionless tube radius

dimensionless distance from wall, $=0.5-R$

distance from wall, $=(d / 2)-r$

dimensionless distance from wall based on wall shear stress

volumetric coefficient of thermal expansion, $1 / \mathrm{K}$

specific heat ratio

thermal conductivity, $\mathrm{W} /(\mathrm{m} \mathrm{K})$

viscosity, Pa s

kinematic viscosity, $\mathrm{m}^{2} / \mathrm{s}$

dimensionless temperature, $=T / T_{w}$

density, $\mathrm{kg} / \mathrm{m}^{3}$

averaged value over the cross-section

effect

Total value

inlet

wall

Reynolds-averaged value

Favre-averaged value

\section{Governing equations}

The problem is formulated by assuming the flow in micro-tube is turbulent and is under constant wall temperature condition. The specific enthalpy is expressed as a function of temperature and pressure by (e.g. Asako, 2016) and is expressed as: 


$$
d h=C_{p} d T+\frac{1-\beta T}{\rho} d p
$$

where $\beta$ is the coefficient of thermal expansion (the isobaric compressibility) and for an ideal gas it is expressed by $\beta=1 / T$. Also, the enthalpy for an ideal gas becomes only a function of $T$ and can be expressed as $\widetilde{h}=C_{p} \widetilde{T}$. The flow is assumed to be steady and axisymmetric. Fluid properties except the density are assumed to be constant. The governing equations can then be expressed as follows:

$$
\begin{aligned}
& \frac{\partial \rho \tilde{u}}{\partial x}+\frac{1}{r} \frac{\partial \rho r \tilde{v}}{\partial r}=0 \\
& \rho \tilde{u} \frac{\partial \tilde{u}}{\partial x}+\rho \tilde{v} \frac{\partial \tilde{u}}{\partial r}=-\frac{\partial p^{*}}{\partial x}+\frac{\partial \tau_{x x}}{\partial x}+\frac{1}{r} \frac{\partial r \tau_{r x}}{\partial r} \\
& \rho \tilde{u} \frac{\partial \tilde{v}}{\partial x}+\rho \tilde{v} \frac{\partial \tilde{v}}{\partial r}=-\frac{\partial p^{*}}{\partial r}+\frac{\partial \tau_{x r}}{\partial x}+\frac{1}{r} \frac{\partial r \tau_{r r}}{\partial r}-\frac{\tau_{\theta \theta}}{r} \\
& \rho C_{p} \tilde{u} \frac{\partial \tilde{T}}{\partial x}+\rho C_{p} \widetilde{v} \frac{\partial \widetilde{T}}{\partial r}=\widetilde{u} \frac{\partial p^{*}}{\partial x}+\widetilde{v} \frac{\partial p^{*}}{\partial r}+\mu \phi+\operatorname{div}\left(\lambda_{\text {eff }} \operatorname{grad} \tilde{T}\right)
\end{aligned}
$$

Where $p^{*}$ is

$$
p^{*}=\bar{p}+\frac{2}{3} \bar{\rho} k
$$

and the viscous stresses are expressed as

$$
\tau_{x x}=2 \mu_{e f f} \frac{\partial \tilde{u}}{\partial x}, \quad \tau_{r r}=2 \mu_{e f f} \frac{\partial \tilde{v}}{\partial r}, \quad \tau_{\theta \theta}=2 \mu_{e f f} \frac{\tilde{v}}{r}, \quad \tau_{x r}=\tau_{r x}=\mu_{e f f}\left(\frac{\partial \tilde{v}}{\partial x}+\frac{\partial \tilde{u}}{\partial r}\right)
$$

$\phi$ is the viscous dissipation function and is expressed as

$$
\phi=2\left\{\left(\frac{\partial \widetilde{u}}{\partial x}\right)^{2}+\left(\frac{\widetilde{v}}{r}\right)^{2}+\left(\frac{\partial \widetilde{v}}{\partial r}\right)^{2}\right\}+\left(\frac{\partial \tilde{u}}{\partial r}+\frac{\partial \widetilde{v}}{\partial x}\right)^{2}
$$

The pressure decreases in the flow direction in the entire region of the tube. Also, the gas expands and the flow accelerates even far from the entrance. However, the changes of velocity and temperature in the flow direction are small compared with the changes in the radial direction. Also, the velocity in the r-direction, $v$, is approximately zero in the region far from the entrance. In the case of a laminar flow, the velocity profile is almost parabolic (Asako et al. 2003). In the case of a turbulent flow, the dimensionless velocity based on the wall shear stress, $u^{+}$, agrees well with the universal velocity profile for an incompressible flow (Murakami and Asako, 2011). Therefore, this region can be considered to be a quasi-fully developed region and it can be assumed that the axial velocity and temperature gradients $(\partial u / \partial x, \partial T / \partial x)$ and $v$ are zero and also the density over the cross-section is constant. The Reynolds number and Mach number can be defined as

$$
\operatorname{Re}=\frac{u_{\text {ave }} d}{\mu / \rho_{\text {ave }}}, M a=\frac{u_{\text {ave }}}{\sqrt{\gamma R_{\text {gas }} T_{w}}}
$$

where $u_{\text {ave }}$ and $\rho_{\text {ave }}$ are the cross-sectional averaged velocity and density as expressed by 


$$
u_{\text {ave }}=\frac{8}{d^{2}} \int_{0}^{d / 2} r \tilde{u} d r, \rho_{\text {ave }}=\int_{0}^{d / 2} \rho r \tilde{u} d r / \int_{0}^{d / 2} r \tilde{u} d r
$$

Note, that the Reynolds number is constant along the tube since it is proportional to the mass flow rate. However, the Mach number which is based on the average velocity of the cross-section increases along the tube because of the increase of the velocity.

The viscous stresses in the quasi-fully developed region are expressed as

$$
\tau_{x x}=0, \quad \tau_{r r}=0, \quad \tau_{\theta \theta}=0, \tau_{x r}=\tau_{r x}=\mu_{e f f}\left(\frac{d \tilde{u}}{d r}\right)
$$

The viscous dissipation function is expressed as

$$
\phi=\left(\frac{d \tilde{u}}{d r}\right)^{2}
$$

Then, Eqs. (2) and (4) become

$$
\begin{aligned}
& 0=-\frac{d \bar{p}}{d x}+\frac{1}{r} \frac{d}{d r}\left(\mu_{e f f} r \frac{d \tilde{u}}{d r}\right) \\
& 0=\tilde{u} \frac{d \bar{p}}{d x}+\mu_{e f f}\left(\frac{d u}{d r}\right)^{2}+\frac{1}{r} \frac{d}{d r}\left(\lambda_{e f f} r \frac{d \tilde{T}}{d r}\right)
\end{aligned}
$$

where the first term on the right hand side of Eq. (14) is the SDP term and the second term is the VD term. Here, the following dimensionless variables are defined:

$$
X=\frac{x}{d}, \quad R=\frac{r}{d}, \quad U=\frac{\widetilde{u}}{u_{\text {ave }}}, \quad P=\frac{\bar{p}}{\rho_{\text {ave }} u_{\text {ave }}^{2}}, \quad \theta=\frac{\widetilde{T}}{T_{w}}
$$

Equations (13) and (14) can be rewritten as

$$
\begin{aligned}
& 0=-\frac{d P}{d X}+\frac{1}{\operatorname{Re}} \frac{1}{R} \frac{d}{d R}\left\{\frac{\mu_{\text {eff }}}{\mu} R \frac{d U}{d R}\right\} \\
& 0=(\gamma-1) M a^{2}\left\{U \frac{d P}{d X}\right\}+(\gamma-1) \frac{M a^{2}}{\operatorname{Re}} \frac{\mu_{\text {eff }}}{\mu}\left(\frac{d U}{d R}\right)^{2}+\frac{1}{\operatorname{RePr}} \frac{1}{R} \frac{d}{d R}\left(R \frac{\lambda_{\text {eff }}}{\lambda} \frac{d \theta}{d R}\right)
\end{aligned}
$$

The boundary conditions are expressed as

$$
\begin{array}{llcl}
R=0 & : & d U / d R=d \theta / d R=0 \\
R=0.5 & : & U=0, \theta=0
\end{array}
$$

Since the correlation between the pressure gradient and the wall shear stress in the quasi-fully developed region for the constant density flow is

$$
\frac{d p}{d x} \frac{\pi}{4} d^{2}=-\tau_{w} \pi d=-C_{f} \frac{1}{2} \rho_{\text {ave }} u_{\text {ave }}^{2} \pi d
$$

Then, the dimensionless pressure gradient can be expressed by the friction coefficient $C_{f}$ as 


$$
\frac{d P}{d X}=-2 C_{f}
$$

Eq. (16) can be rewritten as

$$
0=2 C_{f}+\frac{1}{\operatorname{Re}} \frac{1}{R} \frac{d}{d R}\left\{\frac{\mu_{e f f}}{\mu} R \frac{d U}{d R}\right\}
$$

The shear stress can be expressed by

$$
\tau=\tau_{\mathrm{w}} \frac{r}{d / 2}=\tau_{\mathrm{w}}\left(1-\frac{y}{d / 2}\right) \text { and } \tau=\mu_{\text {eff }} \frac{d u}{d r}
$$

where $y$ is the distance from the wall and is expressed as $y=(d / 2)-r$. Using Eq. (22), $\mu_{\text {eff }} / \mu$ can be expressed by

$$
\frac{\mu_{e f f}}{\mu}=1+\frac{\mu_{t}}{\mu}=\left(1-\frac{y^{+}}{r_{0}^{+}}\right) \frac{d y^{+}}{d u^{+}}
$$

where $u^{+}=\frac{u}{\sqrt{\tau_{w} / \rho_{\text {ave }}}}, y^{+}=\frac{y \sqrt{\tau_{w} / \rho_{\text {ave }}}}{v}, r_{0}^{+}=\frac{(d / 2) \sqrt{\tau_{w} / \rho_{\text {ave }}}}{v}$

A simple universal velocity profile by Von Kármán (e.g. Mills, 1992) is selected and is expressed as

$$
\begin{array}{lc}
u^{+}=y^{+} & \left(y^{+} \leq 5\right) \\
u^{+}=5 \ln \left(y^{+}\right)-3.05 & \left(5 \leq y^{+} \leq 30\right) \\
u^{+}=2.5 \ln \left(y^{+}\right)+5.5 & \left(y^{+} \geq 30\right)
\end{array}
$$

Then, the derivatives can be expressed by:

$$
\begin{array}{lrl}
d u^{+} / d y^{+}=1 & \left(y^{+} \leq 5\right) \\
d u^{+} / d y^{+}=5 / y^{+} & \left(5 \leq y^{+} \leq 30\right) \\
d u^{+} / d y^{+}=2.5 / y^{+} & \left(y^{+} \geq 30\right)
\end{array}
$$

Since $R e=u_{\text {ave }}^{+}\left(2 r_{0}^{+}\right)$and $u_{\text {ave }}^{+}=\frac{\int_{0}^{r_{0}^{+}} u^{+} r^{+} d r^{+}}{\int_{0}^{r_{0}^{+}} r^{+} d r^{+}}$, the following correlation can be derived:

$$
R e=4 \int_{0}^{r_{0}^{+}} u^{+} d y^{+}-\frac{4}{r_{0}^{+}} \int_{0}^{r_{0}^{+}} y^{+} u^{+} d y^{+}
$$

Substituting Eq. (25) into Eq. (27), then we obtain 


$$
R e=-300.58+\frac{2292.8}{r_{0}^{+}}+5 r_{0}^{+} \ln \left(r_{0}^{+}\right)+3.5 r_{0}^{+}
$$

The wall shear stress is expressed as

$$
\tau_{w}=C_{f} \frac{1}{2} \rho_{\text {ave }} u_{\text {ave }}^{2}
$$

Substituting Eq. (29) into Eq. (24), then we obtain

$$
u^{+}=\frac{U}{\sqrt{C_{f} / 2}}, y^{+}=Y \operatorname{Re} \sqrt{C_{f} / 2}, r_{0}^{+}=0.5 \operatorname{Re} \sqrt{C_{f} / 2}
$$

The following equation is derived from Eq. (30).

$$
C_{f}=8\left(\frac{r_{0}^{+}}{R e}\right)^{2}
$$

Substituting Eq. (30) into Eq. (25), we obtain the velocity profile as

$$
\begin{aligned}
& U=4 \frac{\left(r_{0}^{+}\right)^{2}}{\operatorname{Re}} Y \\
& U=\left(\frac{r_{0}^{+}}{R e}\right)\left\{10 \ln \left(2 r_{0}^{+} Y\right)-6.1\right\} \\
& U=\left(\frac{r_{0}^{+}}{\operatorname{Re}}\right)\left\{5 \ln \left(2 r_{0}^{+} Y\right)+11\right\} \\
& \left(y^{+} \leq 5\right) \\
& \left(5 \leq y^{+} \leq 30\right) \\
& \left(y^{+} \geq 30\right)
\end{aligned}
$$

Note that Eq. (32) expresses the velocity profile in the quasi-fully developed region. So, we don't need to solve Eq. (21) to obtain the $U$.

Substituting Eqs. (26) and (30) into Eq. (23), we obtain

$$
\begin{array}{lr}
\frac{\mu_{\text {eff }}}{\mu}=1+\frac{\mu_{t}}{\mu}=\left(1-\frac{Y}{0.5}\right) & \left(y^{+} \leq 5\right) \\
\frac{\mu_{\text {eff }}}{\mu}=1+\frac{\mu_{t}}{\mu}=\left(1-\frac{Y}{0.5}\right) Y \frac{\operatorname{Re} \sqrt{C_{f} / 2}}{5} & \left(5 \leq y^{+} \leq 30\right) \\
\frac{\mu_{\text {eff }}}{\mu}=1+\frac{\mu_{t}}{\mu}=\left(1-\frac{Y}{0.5}\right) Y \frac{\operatorname{Re} \sqrt{C_{f} / 2}}{2.5} & \left(y^{+} \geq 30\right)
\end{array}
$$

The effective thermal conductivity is expressed as

$$
\frac{\lambda_{e f f}}{\lambda}=\frac{\lambda+\lambda_{t}}{\lambda}=1+\frac{\lambda_{t}}{\lambda}=1+\frac{\mu_{t} / P r_{t}}{\mu / P r}
$$

where $\operatorname{Pr}$ is the Prandtl number and $P r_{t}$ is the turbulent Prandtl number and their values are, respectively, chosen as 0.7 and 0.9 . The turbulent viscosity is easily obtained from Eq. (33) as 


$$
\begin{aligned}
\frac{\mu_{t}}{\mu}=\left(1-\frac{Y}{0.5}\right)-1 & \left(y^{+} \leq 5\right) \\
\frac{\mu_{t}}{\mu}=\left(1-\frac{Y}{0.5}\right) Y \frac{\operatorname{Re} \sqrt{C_{f} / 2}}{5}-1 & \left(5 \leq y^{+} \leq 30\right) \\
\frac{\mu_{t}}{\mu}=\left(1-\frac{Y}{0.5}\right) Y \frac{\operatorname{Re} \sqrt{C_{f} / 2}}{2.5}-1 & \left(y^{+} \geq 30\right)
\end{aligned}
$$

Substituting Eq. (35) into Eq. (34), the effective thermal conductivity is obtained as

$$
\begin{array}{ll}
\frac{\lambda_{\text {eff }}}{\lambda}=1+\left\{\left(1-\frac{Y}{0.5}\right)-1\right\} \frac{\operatorname{Pr}}{P r_{t}} & \left(y^{+} \leq 5\right) \\
\frac{\lambda_{\text {eff }}}{\lambda}=1+\left\{\left(1-\frac{Y}{0.5}\right) Y \frac{\operatorname{Re} \sqrt{C_{f} / 2}}{5}-1\right\} \frac{\operatorname{Pr}}{\operatorname{Pr}} & \left(5 \leq y^{+} \leq 30\right) \\
\frac{\lambda_{\text {eff }}}{\lambda}=1+\left\{\left(1-\frac{Y}{0.5}\right) Y \frac{\operatorname{Re} \sqrt{C_{f} / 2}}{2.5}-1\right\} \frac{\operatorname{Pr}}{\operatorname{Pr}} & \left(y^{+} \geq 30\right)
\end{array}
$$

The temperature profile in the quasi-fully developed region can be obtained by solving Eq. (17) with Eqs. (35) \& (36) and with the boundary condition of $\theta=1$ at the wall and $d \theta / d R=0$ at the center.

\section{Numerical computation}

The problem to be solved reduces to a one-dimensional steady diffusion problem. The discretized procedure of the equations is based on the control volume method (Patankar, 1980). A piecewise-linear profile is assumed to evaluate the gradients at the control volume interface. The grid points in the region where $y^{+}<5$ are distributed

\begin{tabular}{|c|c|c|c|c|c|c|}
\hline \multirow{2}{*}{$\begin{array}{c}\text { Grid } \\
\text { points in } \\
y^{+}<5\end{array}$} & \multirow{2}{*}{$\begin{array}{c}\text { Grid } \\
\text { points in } \\
y^{+}>5\end{array}$} & \multirow{2}{*}{$\begin{array}{c}\text { Index of } \\
\text { power-law } \\
\text { spacing }\end{array}$} & \multicolumn{2}{|c|}{$M a=0.2$} & \multicolumn{2}{|c|}{$M a=0.8$} \\
\hline & & & $\theta_{R=0}$ & $\theta_{\text {ave }}$ & $\theta_{R=0}$ & $\theta_{\text {ave }}$ \\
\hline 10 & 50 & 1.5 & $0.98987(-0.03 \%)$ & $0.99249(-0.03 \%)$ & $0.83799(-0.63 \%)$ & $0.87980(-0.59 \%)$ \\
\hline 10 & 100 & 1.5 & $0.99031(0.01 \%)$ & $0.99292(0.01 \%)$ & $0.84502(0.20 \%)$ & $0.88672(0.19 \%)$ \\
\hline 10 & 200 & 1.5 & $0.99010(-0.01 \%)$ & $0.99271(-0.01 \%)$ & $0.84160(-0.20 \%)$ & $0.88333(-0.19 \%)$ \\
\hline 10 & 400 & 1.5 & 0.99021 & 0.99281 & 0.84330 & 0.88501 \\
\hline 5 & 400 & 1.5 & $0.99021(0 \%)$ & $0.99281(0 \%)$ & $0.84331(0.001 \%)$ & $0.88501(0 \%)$ \\
\hline 10 & 400 & 1.5 & 0.99021 & 0.99281 & 0.84330 & 0.88501 \\
\hline 20 & 400 & 1.5 & $0.99021(0 \%)$ & $0.99281(0 \%)$ & $0.84330(0 \%)$ & $0.88501(0 \%)$ \\
\hline 10 & 400 & 1 & $0.99018(-0.003 \%)$ & $0.99278(-0.003 \%)$ & $0.84283(-0.06 \%)$ & $0.88454(-0.05 \%)$ \\
\hline 10 & 400 & 1.5 & 0.99021 & 0.99281 & 0.84330 & 0.88501 \\
\hline 10 & 400 & 2 & $0.99020(-0.001 \%)$ & $0.99280(-0.001 \%)$ & $0.84315(-0.02 \%)$ & $0.88486(-0.02 \%)$ \\
\hline
\end{tabular}
uniformly and non-uniformly where $y^{+}>5$ with higher concentration of grids closer to $y^{+}=5$. The grid size gradually increased from $y^{+}=5$ by the power-law expression (Patankar, 1991). All computations were performed under 10 grid points in the region where $y^{+}<5$ and 400 grid points in the region where $y^{+}>5$. The selected index of the power-law spacing was 1.5 for all the cases.

Table 1 Grid size effect $\left(R e=5 \times 10^{4}\right)$ 
The grid size effect on the dimensionless temperature has been examined for $M a=0.2$ and 0.8 . Other parameters are $R e=50000, \operatorname{Pr}=0.7, \operatorname{Pr}_{t}=0.9$ and $\gamma=1.4$. The dimensionless temperature at the centerline $(R=0)$ and the dimensionless averaged temperature in the cross-section were obtained under various grid points, are listed in Table 1. Comparisons of the $\theta_{R=0}$ and $\theta_{\text {ave }}$ with values obtained under 400 grid points in the region where $y^{+}>5$ are also shown in Table 1. The grid size effect in the region where $y^{+}<5$ is small. Finally, a combination of 10 grid points in $y^{+}<5$, 400 grid points in $y^{+}>5$ and 1.5 for the index of the power-law spacing was chosen for all the computations.

\section{Results and discussion}

The dimensionless static temperature profiles for $R e=10^{5}$ with the Mach number as the curve parameter are plotted in Fig. 1. In the quasi-fully developed region, the dimensionless static temperature is not unity when the terms SDP and $\mathrm{VD}$ are included in the energy equation. The energy equation including the SDP and VD terms conserves sum of the thermal energy and the kinetic energy (e.g. Asako, 2014). This is the reason why the static temperature decreases when Mach number is high. It is noteworthy that the gas accelerates in the quasi-fully developed region up to $M a \approx 1$ and, therefore, the dimensionless static temperature changes in this region.

The gradient of the static temperature at the wall should be zero in the quasi-fully developed region. However, the temperature gradient at the wall seems to be non-zero. The static temperature profiles near the wall are plotted in Fig. 2. As seen in the figure, the temperature gradient is zero at the wall for all cases. Since the three layers universal velocity profile by Von Kármán is used in this analysis, the temperature profile is not smooth at the interface of the layers.

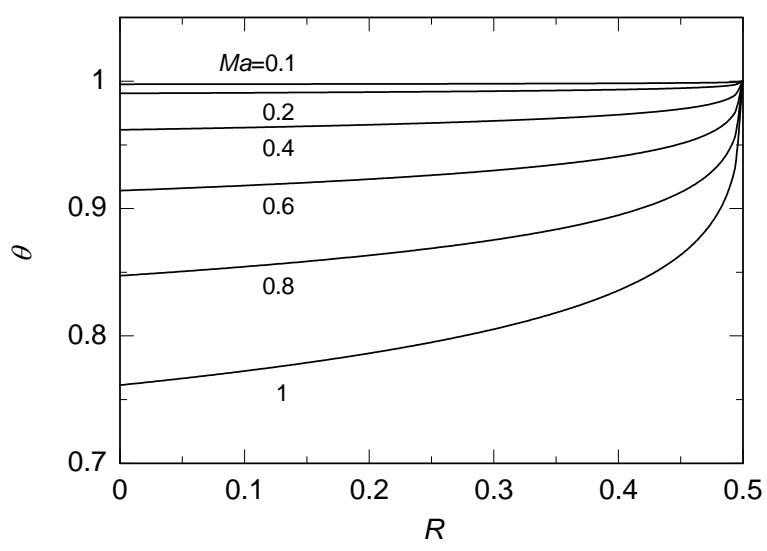

Fig. 1 Temperature profile $\left(R e=10^{5}\right)$

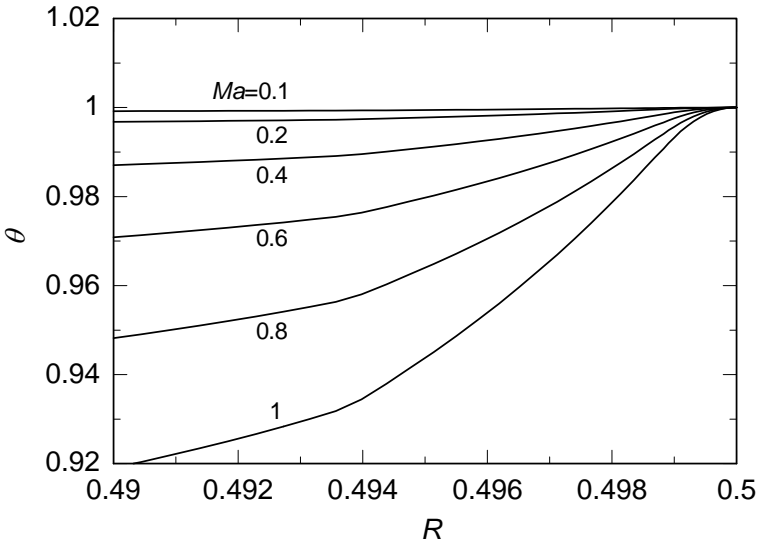

Fig. 2 Temperature profile near wall $\left(R e=10^{5}\right)$

The temperature profiles for an ideal gas flow in a tube of $500 \mu \mathrm{m}$ in diameter and $0.05 \mathrm{~m}$ in length have been numerically obtained in our previous study (Kawashima et al., 2016). The numerical methodology is based on the arbitrary Lagrangian-Eulerian method using the Lam-Bremhorst low Reynolds number (LB1) as the turbulence model (Patel et al., 1984). The compressible momentum and energy equations with the assumption of the ideal gas were solved using the Reynolds number of $1.5 \times 10^{4}$. The temperature profiles of the present study were compared with previous results in Fig. 3. A slight discrepancy is observed even though the agreement is good. The assumptions of the quasi-fully developed region of the present study are applicable for a gas flow in a micro-tube.

To compare the temperature profile of the present study with laminar flow results, both the temperature profiles are plotted in Fig. 4. The temperature profile of the laminar flow is easily obtained from the energy equation using the parabolic velocity profile as is expressed by Asako and Hong, 2017.

$$
\theta=1-2(\gamma-1) M a^{2} \operatorname{Pr}\left(4 R^{2}-1\right)^{2}
$$


Because the velocity profile for turbulent flow is flatter than that of laminar flow, the temperature profile of the turbulent flow is also flatter than that of laminar flow.

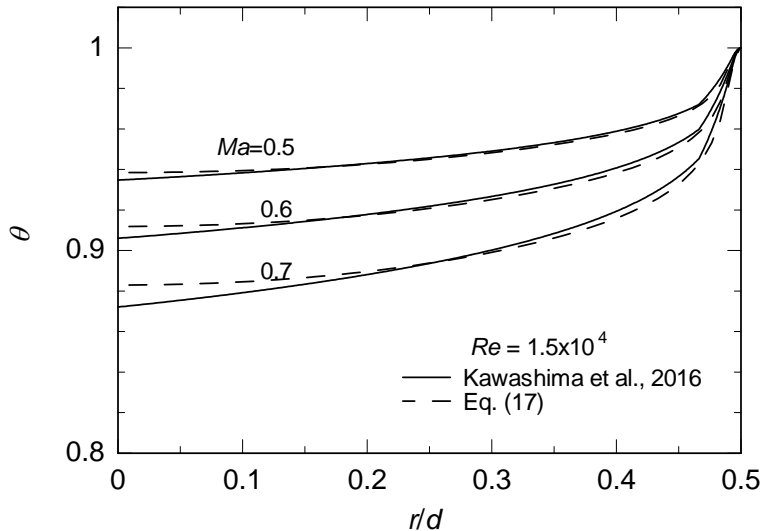

Fig. 3 Comparisons of $\theta$ with previous results

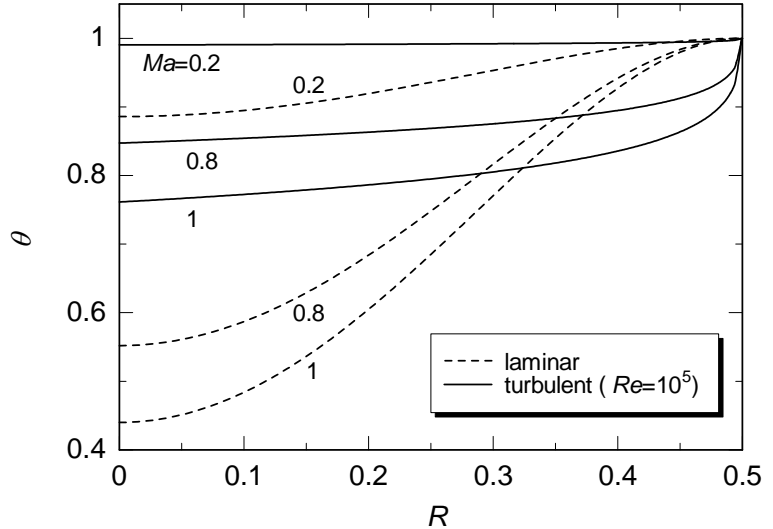

Fig. 4 Comparisons of $\theta$ with that of laminar flow

The total temperature is the sum of the static and kinetic temperatures and is expressed as

$$
T_{T}=T+\frac{u^{2}}{2 C_{p}}
$$

Substituting Eq. (15) into Eq. (38), the dimensionless total temperature, $\theta_{T}=T_{T} / T_{w}$, is obtained as

$$
\theta_{T}=\theta+\frac{\gamma-1}{2} U^{2} M a^{2}
$$

Note that $T_{T}$ and $\theta_{T}$ are not the averaged temperatures. Only the Mach number, $M a$, is averaged over the cross-section. The dimensionless total temperature profiles of $R e=10^{5}$ are plotted in Fig. 5 with the Mach number as the curve parameter. The total temperature is slightly higher than the wall temperature. The static temperature at the center of the tube takes the lowest value; however, the total temperature at the center of the tube is the highest. The dimensionless total temperature profiles near the wall are plotted in Fig. 6. As seen in the figure, the slight discontinuity of the total temperature at $R=0.4937$ is observed. The correlation between $y^{+}$and $r_{0}{ }^{+}$from Eq. (30) can be expressed as

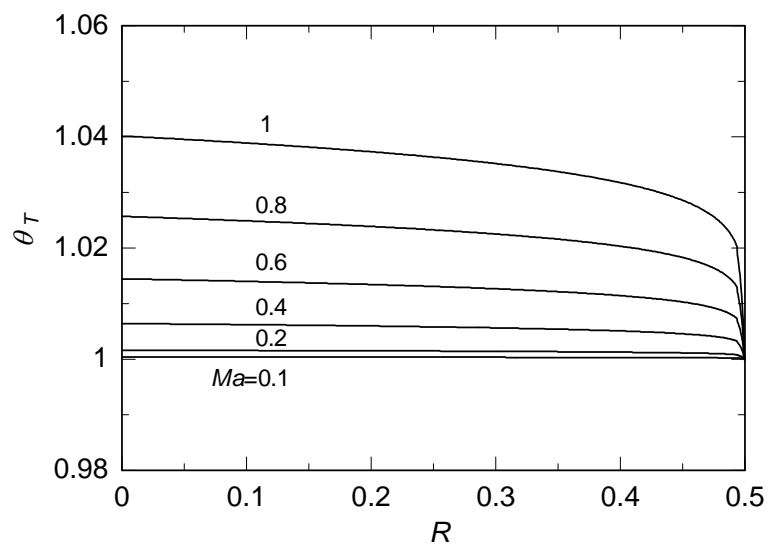

Fig. 5 Total temperature profile $\left(R e=10^{5}\right)$

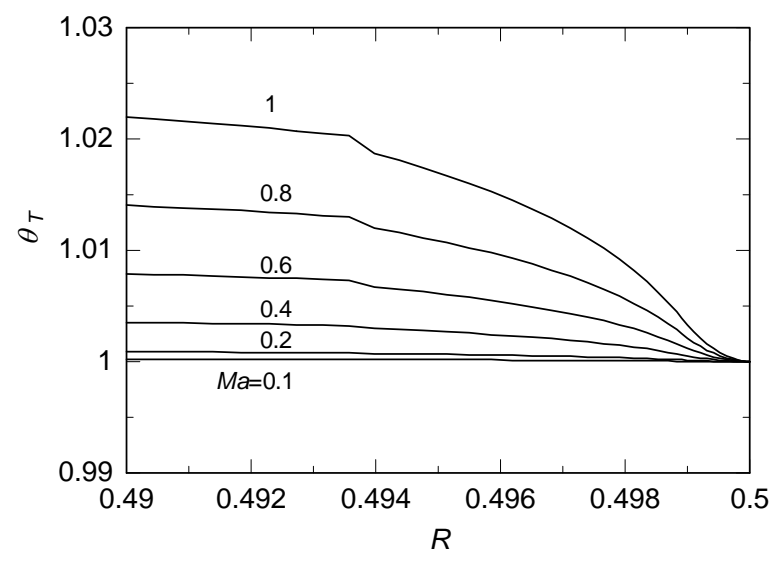

Fig. 6 Total temperature profile near wall $\left(R e=10^{5}\right)$ 


$$
y^{+}=2 Y r_{0}^{+}=2(0.5-R) r_{0}^{+}
$$

Eq. (28) expresses the correlation between $R e$ and ${r_{0}}^{+}$where $r_{0}{ }^{+}$takes a value of 2368.36 when $R e=10^{5}$. Substituting $R$ $=0.4937$ and $r_{0}{ }^{+}=2368.36$ into Eq. (40), $y^{+}=30$ is obtained. Therefore, the slight discontinuity of the total temperature occurs at around $y^{+}=30$. The total temperature gradient is zero at the wall for all cases becasue the fluid velocity near the wall is low and the effect of the kinetic energy on the total temperature is small.

The averaged static temperature and the averaged total temperature in the cross-section are defined as

$$
T_{\text {ave }}=\int_{0}^{d / 2} \rho r \tilde{u} \widetilde{T} d r / \int_{0}^{d / 2} \rho r \tilde{u} d r, T_{T, a v e}=\int_{0}^{d / 2} \rho r \tilde{u} \widetilde{T}_{T} d r / \int_{0}^{d / 2} \rho r \tilde{u} d r
$$

The dimensionless averaged static temperature and the dimensionless total temperature are defined as

$$
\theta_{\text {ave }}=T_{\text {ave }} / T_{w}, \quad \theta_{T, \text { ave }}=T_{T, \text { ave }} / T_{w}
$$

To compare the dimensionless averaged temperatures of the present study with the results obtained by Kawashima et al. (2016), both results are plotted in Fig. 7. Both the averaged static and total temperatures of the present study are slightly higher than the results obtained by Kawashima et al. (2016).

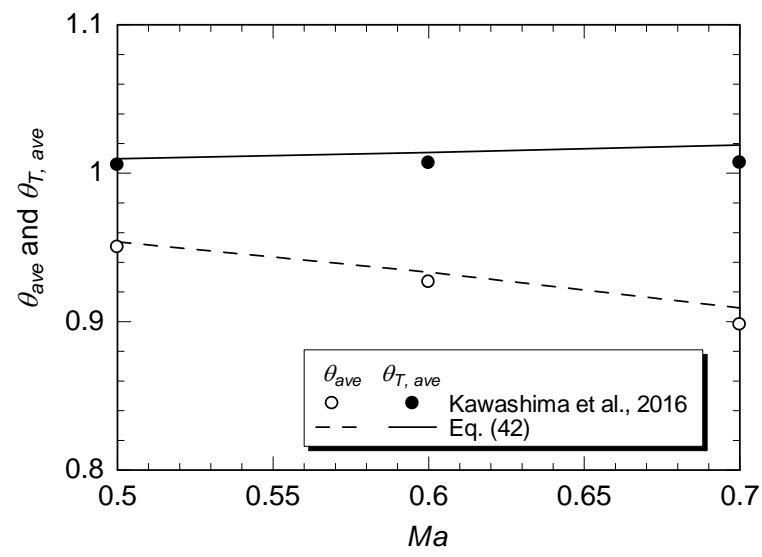

Fig. 7 Comparisons of $\theta_{\text {ave }}$ and $\theta_{T \text {, ave }}$ with previous results

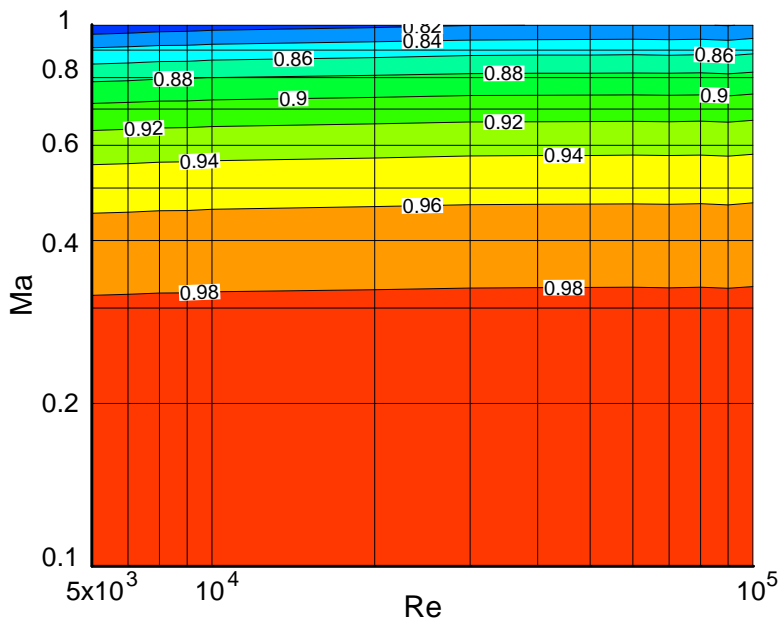

Fig. 8 Dimensionless average static temperature, $\theta_{\text {ave }}$

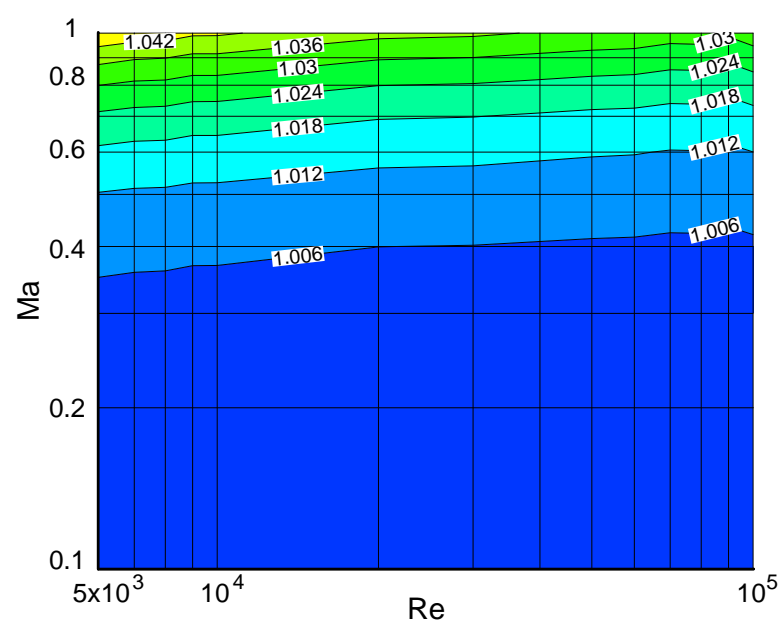

Fig. 9 Dimensionless average total temperature, $\theta_{T, a v e}$ 
The contour of the dimensionless averaged static temperature is plotted in Fig. 8. The averaged static temperature is a function of Mach number and it decreases with increasing Mach number. The contour of the dimensionless averaged total temperature is plotted in Fig. 9. The increment of the total temperatures from the inlet to the outlet represents the heat transfer rate from the tube wall. Therefore, if the exit of the tube is located in the quasi-fully developed region and the inlet velocity is uniform, the heat transfer rate can be obtained from the following equation:

$$
\dot{Q}=\dot{m} C_{p}\left(T_{T, a v e}-T_{T, \text { in }}\right)=\dot{m} C_{p}\left\{\theta_{T, \text { ave }} T_{w}-\left(T_{\text {in }}+\frac{\gamma-1}{2} M a_{\text {in }}^{2} T_{w}\right)\right\}
$$

where $\dot{m}$ is the mass flow rate in the tube and $T_{T, \text { in }}$ is the total temperature at the inlet.

\section{Conclusion}

The static and total temperature profiles for turbulent flows in the quasi-fully developed region were obtained by solving the energy equation which includes the SDP and the VD terms. The three-layer universal velocity model of Von Kármán was used for the velocity profile. The following conclusions are reached:

(1) The static temperature in the quasi-fully developed region varies and is not uniform. The static temperature in the core region decreases due to the energy conversion from the thermal energy to the kinetic energy.

(2) The static temperature in the quasi-fully developed region agrees well with the temperature for an ideal gas flow obtained by solving Navier-Stokes and energy equations. The assumptions of the quasi-fully developed region of the present study are applicable for a gas flow in a micro-tube.

(3) The averaged total temperature is slightly higher than the wall temperature, but it is almost equal to the wall temperature.

(4) The heat transfer rate can be obtained from Eq. (43) and Fig. 9, when the tube exit is located in the quasi-fully developed region and the inlet velocity is uniform.

\section{References:}

Asako, Y., Pi, T., Turner, S. E. and Faghri, M., Effect of compressibility on gaseous flows in micro-channels, International Journal of Heat and Mass Transfer, 46 (2003), pp. 3041-3050.

Asako, Y., Notes on Energy Equation and 1st law of Thermodynamics (2014), Kindle e-book (ASIN: B00PGS516M).

Asako, Y., Energy equation of gas flow with low velocity in a micro-channel, Journal of Heat Transfer, Vol. 138, No. 4 (2016), pp. 041702-1-041702-5.

Asako, Y. and Hong, C., On temperature jump condition of slip flow in a micro-channel with constant wall temperature, Journal of Heat Transfer, Vol. 139, No. 7 (2017), pp. 072402-1-072402-7 .

Kawashima, D., Yamada, T, Hong, C. and Asako, Y., Mach number at outlet plane of a straight micro-tube, Proceeding of IMechE Part C: Journal of Mechanical Engineering Science, Vol. 230, No. 19 (2016), pp. 3420-3430.

Mills, A.F., Heat transfer (1992), p. 432, Irwin.

Patankar, S.V., Numerical Heat Transfer and Fluid Flow (1980), p. 30, Hemisphere Publishing Corp.

Patankar, S.V., Computation of Conduction and Duct Flow Heat Transfer (1991), Innovative Research, Inc. 
Patel, V.C., Rodi, W. and Scheuerer, G., Turbulence models for near-wall and low Reynolds number flows: A review, AIAA Journal, vol. 23, No. 9 (1984), pp. 1308-1319.

Von Kármán T., The analogy between fluid friction and heat transfer, Transactions of the ASME 61 (1939), pp. 705-710. 